\title{
BMJ Open How common is isolated dysphasia among patients with stroke treated with intravenous thrombolysis, and what is their outcome? Results from the SITS-ISTR
}

\author{
Erik Lundström, ${ }^{1}$ Andrea Zini, ${ }^{2}$ Nils Wahlgren, ${ }^{1}$ Niaz Ahmed ${ }^{1}$
}

\begin{abstract}
To cite: Lundström E, Zini A, Wahlgren $\mathrm{N}$, et al. How common is isolated dysphasia among patients with stroke treated with intravenous thrombolysis, and what is their outcome? Results from the SITS-ISTR. BMJ Open 2015;5:e009109. doi:10.1136/bmjopen-2015009109
\end{abstract}

- Prepublication history for this paper is available online. To view these files please visit the journal online (http://dx.doi.org/10.1136/ bmjopen-2015-009109).

Received 16 June 2015 Revised 3 September 2015 Accepted 22 October 2015

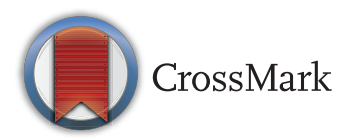

${ }^{1}$ Department of Neurology, Karolinska University Hospital \& Department of Clinical Neuroscience, Karolinska Institutet, Stockholm, Sweden

${ }^{2}$ Stroke Unit, Department of Neuroscience, Nuovo Ospedale Civile "S AgostinoEstense," AUSL Modena, Modena, Italy

Correspondence to Dr Erik Lundström; erik.lundstrom@ki.se

\section{ABSTRACT}

Objectives: To describe the frequency and outcome of isolated dysphasia among patients treated with intravenous thrombolysis (IVT).

Design: Patients registered in the SITS International Stroke Thrombolysis Register (SITS-ISTR).

Participants: Patients with stroke $(\mathrm{N}=58$ 293) treated with IVT between December 2002 and December 2012.

Setting: A multinational, prospective, observational monitoring register.

Main outcome measures: Isolated dysphasia and modified Rankin Scale (mRS).

Methods: We identified patients presenting with isolated dysphasia by reviewing items within the baseline National Institutes of Health Stroke Scale (NIHSS). We performed descriptive statistics for baseline and demographic data, and reported patients' characteristics, radiological data and changes in their NIHSS score within 7 days and mRS score at 3 months. We also reported corresponding data from the general SITS-ISTR cohort.

Results: We found isolated dysphasia at baseline in $1.14 \%$ (663/58 293) of all patients treated with IVT patients. Patients with isolated dysphasia had a longer onset to treatment time, lower proportion of visible infarctions on admission imaging scan and atrial fibrillation, and were less often classified as having large vessels causing strokes, in comparison with the rest of the SITS-ISTR. Symptomatic intracerebral haemorrhage occurred in $2.3 \%$ of patients per SITS-MOST definition and fatal outcome in $5.5 \%$. At 7 days, $50 \%$ of patients with isolated dysphasia recovered completely and at 3 months, $86.3 \%$ patients were functionally independent (mRS score 0-2), $71.7 \%$ had an excellent outcome (mRS score $0-1$ ) and $45.5 \%$ had an mRS score of 0 . Conclusions: A low proportion of patients with isolated dysphasia are treated with IVT. Half of these patients were fully recovered at 7 days.

\section{INTRODUCTION}

Stroke is an important cause of morbidity, with dysphasia as one of the most devastating

\section{Strengths and limitations of this study}

- To the best of our knowledge, this is the largest study of patients with ischaemic stroke with isolated dysphasia treated with intravenous thrombolysis (IVT).

- We identified dysphasia using relevant scores on the National Institutes of Health Stroke Scale (NIHSS), although this method may be criticised as lacking in sensitivity; when including patients in the hyperacute phase, the use of a formal dysphasia battery is practically impossible.

- The study is observational and based on a retrospective analysis of an ongoing database, with all the limitations of this type of study design.

- Another major limitation is the lack of a control group of patients with untreated dysphasia.

symptoms. ${ }^{1}$ Isolated dysphasia gives only up to three points on the National Institutes of Health Stroke Scale (NIHSS) ${ }^{2}$ score, albeit additional points may be given for loss of orientation, and doctors might hesitate to prescribe thrombolysis to patients with low NIHSS scores, due to potential risk of intracranial haemorrhage. ${ }^{3}$ Current guidelines lack the recommendation of whether to treat stroke with low NIHSS score or not, ${ }^{4}$ and approximately $30 \%$ of these patients are not treated with intravenous thrombolysis (IVT). ${ }^{6} 7$

Our knowledge about the incidence and prognosis of isolated dysphasia is sparse. Most of our information is from case series. ${ }^{8-14}$ These studies identify the rate of isolated dysphasia as between $2.4 \%$ and $7.5 \% .^{12-14}$ There are, however, very limited safety and outcome data of isolated dysphasia following intravenous thrombolysis.

We aimed to describe the frequency and outcome of isolated dysphasia among patients treated with IVT, as documented in 
the Safe Implementation of Treatment in StrokeInternational Stroke Thrombolysis Register (SITS-ISTR). We also presented the rest of the SITS-ISTR cohort for an overview.

\section{METHODS}

The SITS-ISTR is a multinational, prospective, observational monitoring register documenting unselected patients with ischaemic stroke treated with IVT. Details of the methods and management can be found elsewhere. ${ }^{15}{ }^{16}$ Between December 2002 and December 2012, 58293 patients treated with IVT were recorded in SITS-ISTR. We searched the SITS register for patients presenting with isolated dysphasia, that is, patients who scored points only on item 9 (Best Language) of NIHSS at baseline. A patient can score a maximum of three points on item 9 . However, it is likely that a patient with dysphasia will score additional points on item $1 \mathrm{~b}$ (Level of Consciousness Questions; maximum 2 points) and item 1c (Level of Consciousness Command; maximum 2 points), achieving up to 7 points for dysphasia in total on the NIHSS. Thus, we defined isolated dysphasia as a score of 1-3 points on item 9 only, with or without points on $1 \mathrm{~b}$ and $1 \mathrm{c}$.

The stroke subtype was classified according to the TOAST criteria. ${ }^{17}$

We assessed early outcomes by the change in the NIHSS score within 7 days after thrombolysis. We reported dysphasia as completely improved if the NIHSS scored 0 on day 7 , partially improved if NIHSS score ranged between 1 and 6 points, and not improved if no change was observed on NIHSS score.

The primary outcome of this study was full recovery, that is, no symptoms at all, excluding also dysphasia (modified Rankin Scale, $\mathrm{mRS}=0$ ) at 3 months. Secondary outcomes were functional independence (mRS score of $\leq 2)$, excellent outcome $(m R S \leq 1)$ and death at 3 months. We defined symptomatic intracerebral haemorrhage $(\mathrm{SICH})$ per the SITS-MOST protocol $^{18}$ as a local or remote parenchymal haemorrhage type 2 on the 22-36 h post-treatment imaging scan or earlier if clinically indicated, combined with a neurological worsening of $\geq 4$ points between baseline and $24 \mathrm{~h}$, or that leading to death. For comparison with other published work, we also report SICH per the National Institute of Neurological Disorders and Stroke (NINDS) definition: ${ }^{19}$ Any intracerebral haemorrhage on any posttreatment imaging scans combined with any decline in neurological status as measured by NIHSS between baseline and 7 days; and SICH per the Second EuropeanAustralasian Acute Stroke Study (ECASS-II) protocol: ${ }^{20}$ Any intracerebral haemorrhage on any post-treatment imaging scans combined with NIHSS worsening $\geq 4$ points between baseline and day 7. All evaluations of imaging studies and neurological status were performed according to clinical routine by the local sites. All definitions of SICH were centrally adjudicated by the SITS
International Coordination Office, based on the clinical and imaging data entered into the registry by the investigators. We also reported the corresponding data from the general SITS-ISTR cohort.

\section{Statistical testing}

We performed descriptive statistics for baseline and demographic data. For categorical variables, we calculated percentage proportions by dividing the number of events by the total number of patients, excluding missing or unknown cases. We did not perform any formal statistical testing between patients with and without isolated dysphasia, since this was not within the primary purpose of the study. We performed all analyses using STATISTICA software V.11.0.

\section{RESULTS}

At baseline, we identified $1.14 \%$ (663/58 293) patients with isolated dysphasia treated with IVT.

Patients with isolated dysphasia had a longer onset to treatment time, lower proportion of visible infarctions on admission imaging scan, lower proportion of atrial fibrillation (AF) and were less often classified as large vessel disease causing strokes, in comparison with the whole SITS-ISTR (table 1).

Within the range 2-7 points on the baseline NIHSS, approximately $45 \%$ of patients improved completely, whereas among patients with a score of 1 on the NIHSS, $75 \%$ completely improved. Only one patient with one point on the NIHSS worsened (table 2).

Of these patient with available data, 50\% (251/505) were completely improved on the NIHSS at day 7 and $45.5 \%(240 / 527)$ had an mRS score of 0 at 3 months, indicating no residual dysphasia (table 3). Altogether, 86.3\% patients were functionally independent (mRS score $0-2$ ) and $71.7 \%$ had an excellent outcome (mRS score $0-1)$ at 3 months. Table 3 shows the outcome at 3 months follow-up for patients with dysphasia, for example, with an initial $1-3$ points on item 9 on the NIHSS (N=527).

Patients with isolated dysphasia had slightly higher rates of SICH according to SITS-MOST definition (2.3\% vs $1.8 \%$, fatal $0.61 \%$ vs $0.29 \%$ ) than the general SITS population but had lower rates of SICH per the ECASS2 $(3.2 \%$ vs $5.3 \%$, fatal $1.3 \%$ vs $2.5 \%)$ and NINDS (5.0\% vs $7.0 \%$, fatal $1.3 \%$ vs $2.5 \%$ ) definitions (table 4 ). For all types of haemorrhages, the percentage was lower in isolated dysphasia than in the SITS-ISTR in general. Table 4 illustrates local haemorrhage at $22-36 \mathrm{~h}$ for patients with dysphasia in comparison with those in the SITS-ISTR.

The mortality was $5.5 \%$ at 3 months compared to $15.6 \%$ in the SITS-ISTR.

The cause of death was known in 26 of 29 cases, and of the 26 cases, the most common causes of death were cerebral infarction $(n=5)$, cerebral haemorrhage $(n=5)$ and pneumonia $(n=3)$; however, one patient died of 
Table 1 Demographic and baseline characteristics of patients

\begin{tabular}{|c|c|c|}
\hline Characteristic & $\begin{array}{l}\text { Isolated dysphasia } \\
(n=663)\end{array}$ & $\begin{array}{l}\text { All patients in the SITS-ISTR } \\
(\mathrm{n}=58293)\end{array}$ \\
\hline Age, years, median (IQR) & $71(62-78)$ & $70(61-77)$ \\
\hline Female, \% & 45.3 & 43.5 \\
\hline OTT, min, median (IQR) & $160(124-190)$ & $149(118-175)$ \\
\hline Baseline NIHSS score, median (IQR) & $4(3-5)$ & $12(7-17)$ \\
\hline No visible infarct signs on admission CT/MRI scan, \% & 15.6 & 21.0 \\
\hline Systolic BP before tPA, mm Hg, median (IQR) & $154(140-168)$ & $150(137-168)$ \\
\hline Diastolic BP before tPA, mm Hg, median (IQR) & $81(74-90)$ & $81(73-90)$ \\
\hline Baseline blood glucose, mmol/L, median (IQR) & $6.3(5.5-7.6)$ & $6.6(5.7-7.9)$ \\
\hline \multicolumn{3}{|l|}{ Medical history and medication, \% } \\
\hline Hypertension & 59.8 & 64.5 \\
\hline Diabetes mellitus & 18.4 & 17.6 \\
\hline Previous stroke & 13.0 & 12.8 \\
\hline Hyperlipidaemia & 31.7 & 33.4 \\
\hline Atrial fibrillation & 19.6 & 24.6 \\
\hline Congestive heart failure & 6.4 & 8.6 \\
\hline Aspirin & 37.0 & 32.5 \\
\hline Other antiplatelet agent & 10.8 & 8.1 \\
\hline Oral antihypertensive & 51.5 & 51.7 \\
\hline Any oral anticoagulation & 2.0 & 2.8 \\
\hline \multicolumn{3}{|l|}{ Aetiology ${ }^{17}$} \\
\hline Large vessel disease & 29.3 & 38.3 \\
\hline Cardiac source & 31.8 & 32.8 \\
\hline Small vessel disease & 10.4 & 10.4 \\
\hline Other determined aetiology & 6.8 & 4.0 \\
\hline Undetermined aetiology & 21.7 & 14.5 \\
\hline
\end{tabular}

myocardial infarction and one died of pulmonary embolism.

Figure 1 illustrates the individual mRS scores at 3 months. Patients with isolated dysphasia had a more favourable prognosis than the SITS-ISTR in general.

\section{DISCUSSION}

To the best of our knowledge, this is the largest study of patients with ischaemic stroke with isolated dysphasia treated with IVT. The proportion of isolated dysphasia in our IVT-treated study cohort is lower $(1.14 \%)$ than those of other studies $(2.4-7.5 \%)$ investigating isolated dysphasia without IVT. ${ }^{13}{ }^{14}$ One reason for this finding may be that patients with isolated dysphasia often do not receive IVT, probably due to uncertainty of the benefit/ risk balance.

Fifty per cent of patients with isolated dysphasia in our study improved completely at day 7 and about $72 \%$ patients had excellent recovery (mRS 0-1) at 3 months, which is comparable with that of Maas et al s ${ }^{12}$ study $(58.8 \%)$ at 6 months. However, it is important to note that the follow-up in our cohort was earlier than in their study and both studies are observational and demographic, and baseline factors may differ. Moreover, comparisons between the studies are difficult because of the

Table 2 The changes in the National Institutes of Health Stroke Scale (NIHSS) score within 7 days after thrombolysis according to baseline NIHSS

\begin{tabular}{|c|c|c|c|c|}
\hline $\begin{array}{l}\text { Baseline NIHSS } \\
(\mathrm{n}=505)\end{array}$ & $\begin{array}{l}\text { Complete improvement } \\
(\mathrm{n}=251 ; 50 \%) \\
\mathrm{n}(\%)\end{array}$ & $\begin{array}{l}\text { Partial improvement } \\
(\mathrm{n}=174 ; 34 \%) \\
\mathrm{n}(\%)\end{array}$ & $\begin{array}{l}\text { No improvement } \\
(\mathrm{n}=28 ; 6 \%) \\
\mathrm{n}(\%)\end{array}$ & $\begin{array}{l}\text { Worsening } \\
(n=52 ; 10 \%) \\
n(\%)\end{array}$ \\
\hline $7(n=30)$ & $14(47)$ & $14(47)$ & $0 /(0)$ & $2 /(7)$ \\
\hline $6(n=82)$ & $36(44)$ & $38(47)$ & $1 /(1)$ & $7 /(9)$ \\
\hline $5(n=104)$ & 49 (47) & 43 (42) & $1 /(1)$ & $11 /(11)$ \\
\hline $4(n=119)$ & $54(45)$ & 40 (33) & $9 /(8)$ & 16/(13) \\
\hline $3(n=56)$ & 32 (57) & 15 (27) & $1 /(2)$ & $8 /(14)$ \\
\hline $2(n=66)$ & $30(45)$ & $24(36)$ & $5 /(8)$ & $7 /(11)$ \\
\hline $1(n=48)$ & $36(75)$ & $0(0)$ & $11 /(23)$ & $1 /(2)$ \\
\hline
\end{tabular}


Table 3 Outcome at 3-month follow-up for patients with dysphasia, for example, with initial 1-3 points on item 9 on the National Institutes of Health Stroke Scale (NIHSS) (N=527)

\begin{tabular}{llllr}
\hline $\begin{array}{l}\text { Modified Rankin } \\
\text { Scale at 3 months }\end{array}$ & $\begin{array}{l}\text { 1 Point on item 9 NIHSS } \\
\text { at baseline (n/N\%) }\end{array}$ & $\begin{array}{l}\text { 2 Points on item 9 NIHSS } \\
\text { at baseline (n/N\%) }\end{array}$ & $\begin{array}{l}\text { 3 Points on item 9 NIHSS } \\
\text { at baseline (n/N\%) }\end{array}$ & All groups \\
\hline 0 & $65(12.3)$ & $148(28.1)$ & $27(5.1)$ & $240(45.5 \%)$ \\
1 & $29(5.5)$ & $96(18.2)$ & $13(2.5)$ & $138(26.2 \%)$ \\
2 & $6(1.1)$ & $64(12.1)$ & $7(1.3)$ & $77(14.6 \%)$ \\
3 & $4(0.8)$ & $15(2.8)$ & $6(1.1)$ & $25(4.7 \%)$ \\
4 & $1(0.2)$ & $11(2.1)$ & $2(0.4)$ & $14(2.7 \%)$ \\
5 & 0 & $4(0.76)$ & 0 & $4(0.8 \%)$ \\
6 & $3(0.6)$ & $22(4.2)$ & $4(0.8)$ & $29(5.5 \%)$ \\
\hline
\end{tabular}

low number of patients with isolated dysphasia $(\mathrm{n}=17)$ in Maas $e t a l$ s study. ${ }^{12}$ In a study by Nesi $e t a l$, the presence of dysphasia was an independent predictor of unfavourable outcome. They observed that patients with dysphasia treated with thrombolysis had a favourable outcome $(83 \%, 10 / 12)$ compared to those who did not receive thrombolysis $(50 \%, 5 / 10)$. However, due to the low number of patients with isolated dysphasia $(n=13)$, it becomes hard to make any certain conclusions from their study.

Dysphasia has a substantial spontaneous improvement within the first months, ${ }^{21-24}$ and stroke severity is correlated to the prognosis. ${ }^{22}{ }^{25}$ We observed a similar proportion of complete improvement as Maas et al observed in their study for those with baseline NIHSS score from 2 to 7.

At 3-months follow-up, $45.5 \%$ of our patients had mRS score of 0 , indicating no remaining dysphasia. Maas et $a l^{12}$ found that nearly twice as many, $85.7 \%(12 / 14)$, recovered at their 6-month follow-up. Unfortunately, there is no further information on the prognosis of isolated dysphasia in the literature. There are several explanations for the discrepancy between our study and that of Maas et al. First, NIHSS is not included in our 3-month follow-up. Second, the mRS is not a measure of language impairment-it is an overall assessment of disability-and it is quite possible that patients with an $m R S=1$ or even $m R S=2$ do not have dysphasia. Another reason is that Maas et al evaluated outcome after 6 months, 3 months later than it was carried out in our study, and further improvement may have occurred within that time period.

As expected, patients with isolated dysphasia differed in several ways from the rest of the SITS cohort. They had less severe stroke and lower proportion of visible infarctions on admission CT scan. The lower proportion of patients with AF and large vessels disease (LVD) in the dysphasic group might be explained by the fact that $\mathrm{AF}$ and LVD are often associated with more severe strokes. ${ }^{26}$

In our study, $86.3 \%$ had a good outcome (mRS 0-2) at 3 months. This proportion is more than $30 \%$ higher than in the SITS-ISTR in general. Lower rates of local as well as remote types of haemorrhage following IVT indicate that this treatment is at least as safe in the isolated dysphasia group as it is in the general SITS-ISTR cohort.

Table 4 Local haemorrhage at 22-36 $\mathrm{h}$ for patients with dysphasia in comparison with the SITS-ISTR

\begin{tabular}{lll}
\hline Local haemorrhages & $\begin{array}{l}\text { Isolated dysphasia (N=641) } \\
\mathbf{n}(\%)\end{array}$ & $\begin{array}{l}\text { All patients in the SITS-ISTR (N=54 993) } \\
\mathbf{n}(\%)\end{array}$ \\
\hline No local haemorrhages & $576(89.9)$ & $47515(86.4)$ \\
$\mathrm{PH} 1$ & $12(1.9)$ & $1518(2.8)$ \\
$\mathrm{PH} 2$ & $16(2.5)$ & $1566(2.9)$ \\
$\mathrm{HI} 1$ & $18(2.8)$ & $2615(4.8)$ \\
HI2 & $19(3.0)$ & $1779(3.2)$ \\
Remote haemorrhages & $\mathrm{N}=642$ & $\mathrm{~N}=54987$ \\
No remote haemorrhages & $625(97.4)$ & $53327(97.0)$ \\
PHr1 & $11(1.7)$ & $1061(1.9)$ \\
PHr2 & $6(0.9)$ & $599(1.1)$ \\
SICH per SITS-MOST & $2.30(15 / 652)$ & $1.84(1022 / 55623)$ \\
SICH per ECASS-2 & $3.2(20 / 632)$ & $5.3(2893 / 54330)$ \\
SICH per NINDS & $5.0(32 / 635)$ & $7.0(3833 / 54450)$ \\
\hline Haemorrhage was classified, using clinical and radiological criteria, as: HI1=small petechiae along the margins of the infarct, HI2=confluent \\
petechiae within the infarcted area but not space-occupying, PH1=blood clots in <30\% of the infarcted area with some slight space-occupying \\
effect, and PH2=blood clot in >30\% of the infarcted area with a substantial space-occupying effect. \\
ECASS-2, Second European-Australasian Acute Stroke Study; NINDS, National Institute of Neurological Disorders and Stroke; SICH, \\
symptomatic intracerebral haemorrhage.
\end{tabular}


Figure 1 The modified Rankin

Scale (mRS) at 3 months for patients with isolated dysphasia and all patients in the SITS International Stroke Thrombolysis Register (SITS-ISTR).

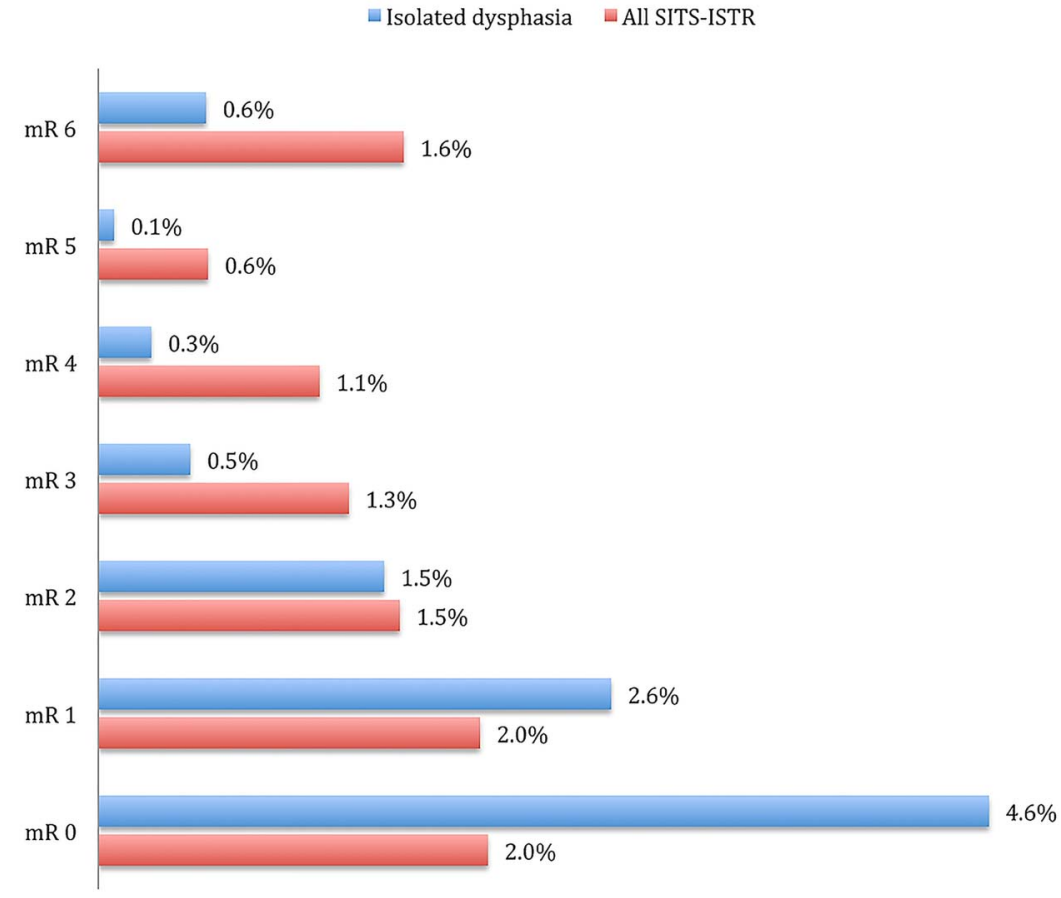

In contrast to the ECASS 2 and NINDS definitions of SICH, the SITS-MOST definition results in slightly higher values for isolated dysphasia. We cannot exclude, however, that the SITS-MOST definition, requiring four points deterioration in combination with a $\mathrm{PH} 2$ or $\mathrm{PH} 2 \mathrm{r}$ type of haemorrhage, may slightly overestimate the risk in patients with a low baseline NIHSS compared to those seen in the general SITS-ISTR population.

We identified dysphasia using relevant scores on the NIHSS. This method may be criticised as lacking in sensitivity. ${ }^{27}$ Many of the previous studies of isolated dysphasia used more sensitive and time-consuming dysphasia batteries. Those studies, ${ }^{8-11}$ however, are case series, and do not include patients in the hyperacute phase, in which the use of a formal dysphasia battery is practically impossible. Consequently, most of the acute stroke trials use item 9 on the NIHSS ${ }^{28}$ as a dysphasia symptom. Our definition of dysphasia was somewhat broader than the previous definitions because we allowed patients to have scores on items $1 \mathrm{~b}$ and $1 \mathrm{c}$ on the NIHSS. This was necessary because patients with dysphasia often give incomplete responses on the consciousness questions and commands.

Our study has several limitations. It is observational and based on a retrospective analysis of an ongoing database, with all the limitations of this type of study design. Another major limitation is the lack of control group of patients with untreated dysphasia. No data from randomised controlled trials on thrombolysis in isolated dysphasia are currently available.

Our strength is that we retrieved the largest cohort of isolated dysphasia treated with IVT.

In conclusion, our study suggests that patients with isolated dysphasia often do not receive IVT. Half of the patients with isolated dysphasia treated with IVT recovered fully within 7 days and $72 \%$ had an excellent outcome at 3 months. The risk of serious haemorrhagic complications and death is low. Considering the solid evidence in favour of IVT, our study suggests that this subgroup of patients should not be treated differently than others.

Contributors NW and NA coordinated the study. NA performed the statistical analysis. EL, NA and NW wrote the initial draft of the manuscript. AZ was local coordinator of a leading recruiting centre. All the authors read and commented on the first draft, with regard to interpretation of the data and editing of the manuscript, and have seen and approved the final version. EL, NA and NW have direct access to the original data, and vouch for the accuracy and completeness of this report.

Funding The SITS-ISTR is funded by an unrestricted grant from Boehringer Ingelheim and Ferrer, and by grants from the EU Public Health Executive Authority, Stockholm County Council and the Karolinska Institute.

Competing interests NA is a senior researcher in SITS International, which receives an unrestricted grant from Boehringer Ingelheim and Ferrer for the SITS-MOST/SITS-ISTR. NW has received expenses from Boehringer Ingelheim for his role as member of the Steering Committee in relation to the ECASS III trial with alteplase and served as a consultant to Thrombogenics as chairman of the DSMB. SITS International (chaired by NW) received a grant from Boehringer Ingelheim and Ferrer for the SITS-MOST/SITS-ISTR. NW has also received lecture fees from Boehringer Ingelheim and Ferrer. This study is a part of the Fighting Stroke Project supported by the Swedish Heart and Lung Foundation and Karolinska Institutet; the project is supported by funding from Friends of Karolinska Institutet, USA, and Johanniterorden. The views expressed are those of the authors.

Ethics approval Ethics approval was obtained in countries that required this; other countries approved the register for conduct as an anonymised audit. The SITS Monitoring Study data (SITS-MOST) are embedded within the SITSEthics Committee of Karolinska Institutet, Stockholm.

Provenance and peer review Not commissioned; externally peer reviewed.

Data sharing statement No additional data are available.

Open Access This is an Open Access article distributed in accordance with the Creative Commons Attribution Non Commercial (CC BY-NC 4.0) license, ISTR. The SITS-MOST, and subsequently the SITS-ISTR, was approved by the 
which permits others to distribute, remix, adapt, build upon this work noncommercially, and license their derivative works on different terms, provided the original work is properly cited and the use is non-commercial. See: http:// creativecommons.org/licenses/by-nc/4.0/

\section{REFERENCES}

1. Gottesman RF, Hillis AE. Predictors and assessment of cognitive dysfunction resulting from ischaemic stroke. Lancet Neurol 2010;9:895-905.

2. Brott $\mathrm{T}$, Marler JR, Olinger $\mathrm{CP}$, et al. Measurements of acute cerebral infarction: lesion size by computed tomography. Stroke 1989;20:871-5.

3. Wardlaw JM, Murray V, Berge E, et al. Recombinant tissue plasminogen activator for acute ischaemic stroke: an updated systematic review and meta-analysis. Lancet 2012;379:2364-72.

4. Jauch EC, Saver JL, Adams HP, et al. Guidelines for the early management of patients with acute ischemic stroke: a guideline for healthcare professionals from the American Heart Association/ American Stroke Association. Stroke 2013;44:870-947.

5. The European Stroke Organisation (ESO) Executive Committee and the ESO Writing Committee. Guidelines for management of ischaemic stroke and transient ischaemic attack 2008. Cerebrovasc Dis 2008;25:457-507.

6. Barber P, Zhang J, Demchuk A, et al. Why are stroke patients excluded from TPA therapy? An analysis of patient eligibility. Neurology 2001:56:1015-20.

7. Khatri P, Kleindorfer DO, Yeatts SD, et al. Strokes with minor symptoms: an exploratory analysis of the National Institute of Neurological Disorders and Stroke recombinant tissue plasminogen activator trials. Stroke 2010;41:2581-6.

8. Deleval J, Leonard A, Mavroudakis N, et al. Global aphasia without hemiparesis following prerolandic infarction. Neurology 1989;39:1532-5.

9. Legatt $A D$, Rubin MJ, Kaplan LR, et al. Global aphasia without hemiparesis: multiple etiologies. Neurology 1987;37:201-5.

10. Tranel D, Biller J, Damasio H, et al. Global aphasia without hemiparesis. Arch Neurol 1987;44:304-8.

11. Ferro JM. Global aphasia without hemiparesis. Neurology 1983;33:1106.

12. Maas MB, Lev MH, Ay $\mathrm{H}$, et al. The prognosis for aphasia in stroke. $J$ Stroke Cerebrovasc Dis 2012;21:350-7.

13. Fennis TF, Compter A, van den Broek MW, et al. Is isolated aphasia a typical presentation of presumed cardioembolic transient ischemic attack or stroke? Cerebrovasc Dis 2013;35:337-40.
14. Giesbers CP, Koehler PJ, Schreuder TH. Is isolated aphasia associated with atrial fibrillation? A prospective study. Cerebrovasc Dis Extra 2014;4:165-73.

15. Ahmed N, Wahlgren N, Grond M, et al. Implementation and outcome of thrombolysis with alteplase 3-4.5 h after an acute stroke: an updated analysis from SITS-ISTR. Lancet Neurol 2010;9:866-74.

16. Wahlgren N, Ahmed N, Dávalos A, et al. Thrombolysis with alteplase $3-4.5 \mathrm{~h}$ after acute ischaemic stroke (SITS-ISTR): an observational study. Lancet 2008;372:1303-9.

17. Adams HP, Bendixen B, Kapelle L, et al. Classification of subtype of acute ischemic stroke. Stroke 1993;24:35-41.

18. Wahlgren N, Ahmed N, Dávalos A, et al. Thrombolysis with alteplase for acute ischaemic stroke in the Safe Implementation of Thrombolysis in Stroke-Monitoring Study (SITS-MOST): an observational study. Lancet 2007;369:275-82.

19. . Tissue plasminogen activator for acute ischemic stroke. The National Institute of Neurological Disorders and Stroke rt-PA Study Group. N Engl J Med 1996;333:1581-7.

20. Larrue $\mathrm{V}$, von Kummer R, Muller A, et al. Risk factors for severe hemorrhagic transformation in ischemic stroke patients treated with recombinant tissue plasminogen activator : a secondary analysis of the European-Australasian Acute Stroke Study (ECASS II). Stroke 2001;32:438-41.

21. Brust JC, Shafer SQ, Richter RW, et al. Aphasia in acute stroke. Stroke 1976;7:167-74.

22. Pedersen PM, Jørgensen HS, Nakayama $\mathrm{H}$, et al. Aphasia in acute stroke: incidence, determinants, and recovery. Ann Neurol 1995;38:659-66.

23. Demeurisse G, Demol O, Derouck M, et al. Quantitative study of the rate of recovery from aphasia due to ischemic stroke. Stroke 1980;11:455-8.

24. Hartman J. Measurement of early spontaneous recovery from aphasia with stroke. Ann Neurol 1981;9:89-91.

25. Kremer C, Perren F, Kappelin J, et al. Prognosis of aphasia in stroke patients early after iv thrombolysis. Clin Neurol Neurosurg 2013;115:289-92.

26. Anderson DC, Kappelle LJ, Eliasziw M, et al. Occurrence of hemispheric and retinal ischemia in atrial fibrillation compared with carotid stenosis. Stroke 2002;33:1963-8.

27. Laska AC, Bartfai A, Hellblom A, et al. Clinical and prognostic properties of standardized and functional aphasia assessments. J Rehabil Med 2007;39:387-92.

28. Ali M, Bath PM, Lyden PD, et al, VISTA Collaboration. Representation of people with aphasia in randomized controlled trials of acute stroke interventions. Int J Stroke 2014;9:174-82. 